\title{
Multimodal Registration using the Discrete Wavelet Frame Transform
}

\author{
Shutao Li $^{1} \quad$ Jinglin Peng ${ }^{1} \quad$ James T. Kwok ${ }^{2} \quad$ Jing Zhang ${ }^{1}$ \\ ${ }^{1}$ College of Electrical and Information Engineering \\ Hunan University \\ Changsha, China \\ ${ }^{2}$ Department of Computer Science \\ Hong Kong University of Science and Technology \\ Clear Water Bay, Hong Kong
}

\begin{abstract}
Image registration is a critical step in medical image analysis. In this paper, a novel image registration method based on the discrete wavelet frame transform (DWFT) and the sum of absolute distance (SAD) method is proposed. First, the multimodal images are decomposed by DWFT, which is shift-invariant compared to traditional dyadic wavelet transforms. Then the energy maps are computed from the details sub-band images. Finally, genetic algorithm (GA) is adopted to obtain the minimum SAD between the two energy maps. The proposed method is tested on 50 pairs of two-dimensional T1-weighted and T2weighted modal images. Experimental results demonstrated that the proposed method can achieve high accuracy in the rigid transformation case.
\end{abstract}

\section{Introduction}

Multimodal image registration finds a geometric relation or transformation between images derived from different modalities. This is an important preprocessing step for other medical image operations. Maintz [6] classified various intrinsic medical image registration methods into three categories: landmark-based, segmentation-based and voxel property-based. As landmark extraction and image segmentation are difficult to perform in some medical images, methods based on voxel properties are more practical and popular $[5,9,15]$.

The wavelet transform is an efficient and powerful tool that has been used to obtain salient geometric characteristics for registration $[1,2,4,8,13,14]$. Many researchers use the wavelet decomposition together with a "coarse to fine" procedure $[1,8,13,14]$ in order to improve the searching speed. In addition to this "coarse to fine" procedure, there are some research works on using the advantages of wavelet sub-bands for feature characterization. For example, Le Moigne et al. used wavelet coefficients with magnitude above $13 \%-15 \%$ of the maximum value to form their feature space [4]. In [2], Dinov et al. applied frequencyadaptive thresholding to the wavelet coefficients in order to keep only the significant coefficients in the wavelet transform domain for registration.

In this paper, a novel multimodal registration method based on the discrete wavelet frame transform (DWFT) is proposed. First, the images are decomposed by DWFT. Then the sub-band energies are computed from three details sub-band images. These reflect more features related to the edges and image details than the decomposed coefficients. The sum of absolute distance (SAD) between the energy maps is selected as the similarity metric. Finally, genetic algorithm is used to search for the minimum SAD, which corresponds to the registration result. The proposed method is validated by simulated magnetic resonance images form the Brainweb.

The paper is organized as follows. A brief introduction to the DWFT is given in section 2. In section 3, the proposed method is described in detail. Experimental setup and results are given in section 4 . In the last section, concluding remarks are given.

\section{Discrete Wavelet Frame Transform}

The wavelet transform is a linear signal transformation using templates $\psi(a, b)=a^{-1 / 2} \psi((x-b) / a)$, which are shifted and dilated versions of a given wavelet function $\psi(x)$ [7]. However, one problem with the commonly used discrete wavelet transform (DWT) is that it is not translation-invariant. To avoid this disadvantage, Unser proposed an overcomplete wavelet representation called the discrete wavelet frame transform (DWFT) [12]. It over- 
comes the shortage of DWT by omitting the down-sampling operations in DWT. Thus, performing DWFT to $d$ decomposition levels will produce a total of $3 d$ details sub-bands and one approximation sub-band of the same size as the original image. On the other hand, in DWT, the images are constructed as a wavelet pyramid. The different decomposed results of these two kinds of transform are illustrated in Figure 1.

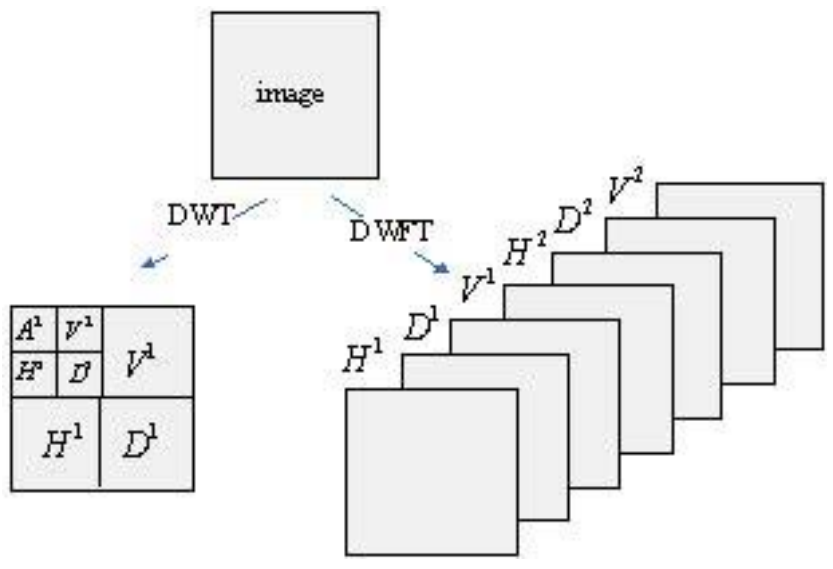

Figure 1. Image decompositions for DWT and DWFT.

\section{The Proposed Method}

A schematic diagram for the proposed registration method is shown in Figure 3. It contains following steps.

Step 1: The reference image $R$ and the floating image $F$ are decomposed by DWFT. Let $\left[A_{R}, H_{R}, V_{R}, D_{R}\right]$ and $\left[A_{F}, H_{F}, V_{F}, D_{F}\right]$ denote the four sub-bands of the reference image and floating image respectively.

Step 2: The energy map at point $(i, j)$ in the image $R$ or $F$ is computed as follows.

$$
E_{I m}=H_{I m}^{2}(i, j)+V_{I m}^{2}(i, j)+D_{I m}^{2}(i, j) .
$$

The energy maps of the simulated human brain (T1, T2weighted magnetic resonance image) using one level of the DWFT decomposition are shown in Figure 2. It can be seen that the energy maps extract most of the salient features of the original images, which will facilitate the registration process.

Step 3: Genetic algorithm [3] is used to search for the optimum registration parameters. The fitness function is the SAD

$$
S A D=\frac{1}{N} \sum_{X_{R} \in \Omega_{F, R}^{\tau}}\left|R\left(X_{R}\right)-F^{\tau}\left(X_{R}\right)\right|,
$$

between the two energy maps of the reference image and the floating image. Here, $R, F$ denote the reference and floating images respectively, $\tau$ is the transformation, $\Omega_{F, R}^{\tau}$ is the overlapped area and $N$ is indexes the pixels in the overlapped area.

\section{Experimental Results}

In the experiments, T1- and T2-weighted MR images $(217 \times 181 \times 1811 \mathrm{~mm}$ cubic voxels $)$ from Brianweb ${ }^{1}$ were used. 50 different slices in the T1-weighted image were selected as reference images. The corresponding T2-weighted slices are transformed by a rigid transformation to form the floating images. For comparison, three other commonlyused registration methods: mutual information(MI) [5], normalized mutual information (NMI) [11], and correlation ratio (CR) [10], are also performed.

The original slices are in 16 bits per pixel. To avoid interpolation artifacts introduced by partial volume interpolation in MI, the images are rescaled to 64 gray levels. This also gives a more obvious maximum in the MI and saves searching time for all the methods. Parameter settings for the genetic algorithm are shown in Table 1.

Table 1. Parameter settings for genetic algorithm.

\begin{tabular}{|l|c|}
\hline & setting \\
\hline number of generations & 50 \\
\hline population & 100, randomly initialized \\
\hline crossover function & intermediate; rate $=1.0$ \\
\hline mutation function & uniform, rate $=0.01$ \\
\hline parameter encoding & real values \\
\hline
\end{tabular}

The "db1" filter is selected as the wavelet basis, because it has the best location accuracy and least computation load compared to the other basis. For a rigid transformation with $4 \mathrm{~mm}$ translation both in $x$-axis and $y$-axis and 4 degree rotation, mean, maximum and median of the registration errors of the 50 slices are listed in Table 2, where the $L$ denotes the decomposition level for DWFT.

From Table 2, we can see that when the decomposition level is one, our proposed method can achieve the smallest mean, maximum, and median of errors in $x$-direction translation. For $y$-direction translation, our method gives a smaller mean of errors than both MI and NMI. For rotation registration, MI and NMI are the best. Our method is just better than CR. From the comparison of results at different decomposition levels, it can be seen that deeper decomposition does not provide better results.

\footnotetext{
${ }^{1}$ http://www.bic.mni.mcgill.ca/brainweb/
} 

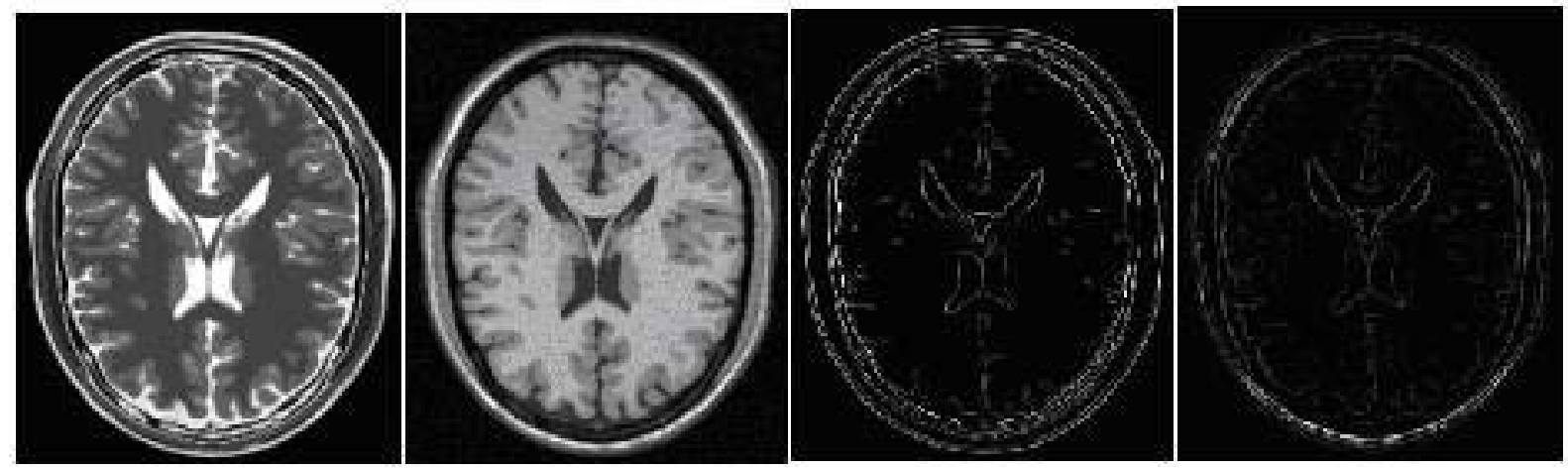

Figure 2. Left: A pair of T1- and T2-wighted images; Right: The corresponding energy maps.

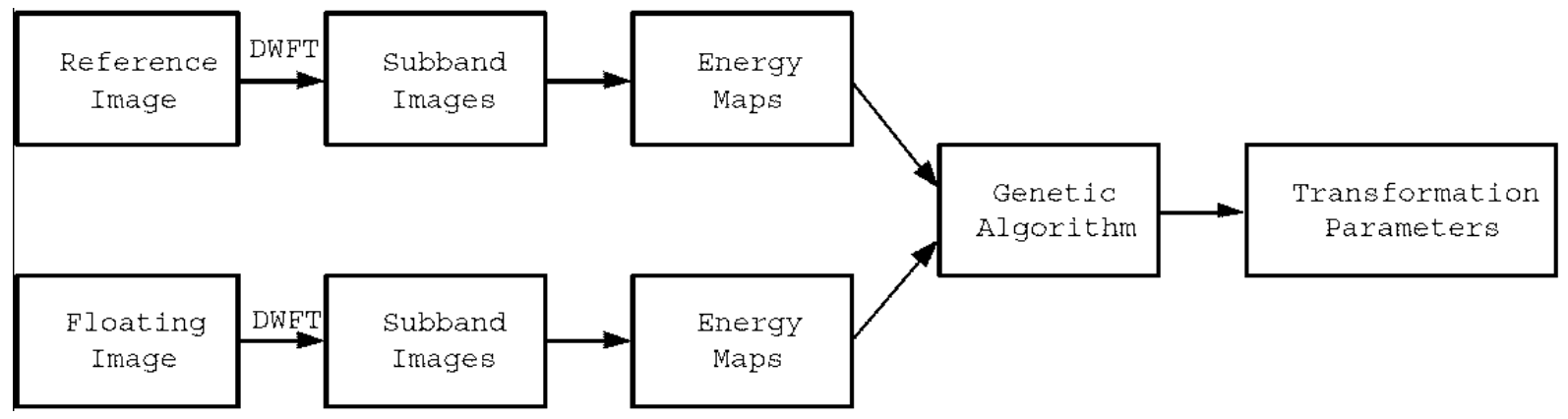

Figure 3. The proposed method.

Table 2. Registration errors.

\begin{tabular}{|c|ccc|ccc|ccc|}
\hline & \multicolumn{3}{|c|}{ mean } & \multicolumn{3}{c|}{ maximum } & \multicolumn{3}{c|}{ variance } \\
& trans- $x$ & trans- $y$ & rotation & trans- $x$ & trans- $y$ & rotation & trans- $x$ & trans- $y$ & rotation \\
\hline our method $(L=1)$ & $\mathbf{0 . 0 3 8 9}$ & 0.2103 & 0.0524 & $\mathbf{0 . 1 7 9 8}$ & 0.4418 & 0.1836 & 0.0012 & 0.0121 & 0.0020 \\
\hline our method $(L=2)$ & 0.1146 & 0.3079 & 0.2590 & 0.3293 & 3.7640 & 2.8065 & 0.0071 & 0.2756 & 0.1501 \\
\hline MI & 0.2348 & 0.3140 & $\mathbf{0 . 0 0 6 7}$ & 0.2504 & 0.3374 & $\mathbf{0 . 0 2 2 2}$ & $\mathbf{0 . 0 0 0 0 5}$ & $\mathbf{0 . 0 0 0 1}$ & $\mathbf{0 . 0 0 0 0 2}$ \\
\hline NMI & 0.2335 & 0.3155 & 0.0071 & 0.2501 & $\mathbf{0 . 3 3 7 1}$ & 0.0272 & 0.00005 & 0.0001 & 0.00003 \\
\hline CR & 0.1376 & $\mathbf{0 . 1 4 4 9}$ & 0.2860 & 0.4777 & 0.4486 & 0.5419 & 0.0160 & 0.0138 & 0.0152 \\
\hline
\end{tabular}




\section{Conclusions}

In this paper, we propose the use of DWFT to obtain the energy maps of the original images. These maps are then registered by SAD, by using GA as the search tool. Experimental results demonstrate that satisfying registration accuracy can be achieved compared to existing techniques. Future work will be done in more complex registration situations.

\section{Acknowledgments}

This work was supported by the National Natural Science Foundation of China (No.60402024) and Program for New Century Excellent Talents in University.

\section{References}

[1] R. Allen, F. Kamangar, and E. Stokely. Laplacian and orthogonal wavelet pyramid decompositions in coarse-tofine registration. IEEE Transactions on Signal Processing, 41(12):3536-3541, 1993.

[2] I. Dinov, M. Mega, P. Thompson, R. Woods, W. de Sumners, E. Sowell, and A. Toga. Quantitative comparison and analysis of brain image registration using frequency-adaptive wavelet shrinkage. IEEE Transactions on Information Technology in Biomedicine, 6(1):73-85, Mar. 2002.

[3] D. Goldberg. Genetic Algorithms in Search, Optimization and Machine Learning. Addison-Wesley Longman, Boston, MA, USA, 1989.

[4] J. Le Moigne, W. Campbell, and R. Cromp. An automated parallel image registration technique based on the correlation of wavelet features. IEEE Transactions on Geoscience and Remote Sensing, 40(8):1849-1864, Aug. 2002.

[5] F. Maes, A. Collignon, D. Vandermeulen, G. Marchal, and P. Suetens. Multimodality image registration by maximization of mutual information. IEEE Transactions on Medical Imaging, 16(2):187-198, 1997.

[6] J. Maintz and M. Viergever. A survey of medical image registration. Medical Image Analysis, 2(1):1-36, 1998.

[7] S. Mallat. A theory for multiresolution signal decomposition: The wavelet representation. IEEE Transactions on Pattern Analysis and Machine Intelligence, 11(7):674-693, July 1989.

[8] H. Own. Image registration algorithm based on complex wavelet transform. International Journal on Graphics, Vision and Image Processing, 2:9-15, 2005.

[9] J. Pluim, J. Maintz, and M. Viergever. Mutual-informationbased registration of medical images: A survey. IEEE Transactions on Medical Imaging, 22(8):986-1004, 2003.

[10] A. Roche, G. Maladain, X. Pennec, and N. Ayache. The correlation ration as a new similarity measure for multimodal image registration. In Proceedings of the International Conference On Medical Image Computing and ComputerAssisted Intervention, pages 1115-1124, 1998.
[11] C. Studholme, D. Hill, and D. Hawkes. An overlap invariant entropy measure of 3D medical image alignment. Pattern Recognition, 32:71-86, 1999.

[12] M. Unser. Texture classification and segmentation using wavelet frames. IEEE Transactions on Image Processing, 4(11):1549-1560, 1995.

[13] M. Unser, P. Thévenaz, C. Lee, and U. Ruttimann. Registration and statistical analysis of PET images using the wavelet transform. IEEE Engineering in Medecine and Biology Magazine, 14(5):603-611, 1995.

[14] J. Wu and A. Chung. Multimodal brain image registration based on wavelet transform using SAD and MI. In Proceedings of the Second International Workshop on Medical Imaging and Augmented Reality, pages 270-277, Beijing, China, Aug. 2004.

[15] B. Zitova and J. Flusser. Image registration methods: A survey. Image and Vision Computing, 21(11):977-1000, 2003. 